\title{
The methanol lines and hot core of OMC2-FIR4, an intermediate-mass protostar, with Herschel/HIFI
}

\author{
M. Kama ${ }^{1}$, C. Dominik ${ }^{1,2}$, S. Maret ${ }^{3}$, F. van der Tak ${ }^{4,5}$, E. Caux ${ }^{6,7}$, C. Ceccarelli ${ }^{3,8,9}$, A. Fuente ${ }^{10}$, N. Crimier ${ }^{3,11}$, \\ S. Lord $^{12}$, A. Bacmann ${ }^{3,8,9}$, A. Baudry ${ }^{8,9}$, T. Bell ${ }^{13}$, M. Benedettini ${ }^{14}$, E. A. Bergin ${ }^{26}$, G. A. Blake ${ }^{13}$, A. Boogert ${ }^{12}$, \\ S. Bottinelli ${ }^{6,7}$, S. Cabrit ${ }^{16}$, P. Caselli ${ }^{15}$, A. Castets ${ }^{3,8,9}$, J. Cernicharo ${ }^{11}$, C. Codella ${ }^{18}$, C. Comito ${ }^{17}$, A. Coutens ${ }^{6,7}$, \\ K. Demyk ${ }^{6,7}$, P. Encrenaz ${ }^{16}$, E. Falgarone ${ }^{16}$, M. Gerin ${ }^{16}$, P. F. Goldsmith ${ }^{19}$, F. Helmich ${ }^{4}$, P. Hennebelle ${ }^{16}$, T. Henning ${ }^{27}$, \\ E. Herbst ${ }^{20}$, P. Hily-Blant ${ }^{3}$, T. Jacq ${ }^{8,9}$, C. Kahane ${ }^{3}$, A. Klotz, ${ }^{6,7}$, W. Langer ${ }^{19}$, B. Lefloch ${ }^{3}$, D. Lis ${ }^{13}$, A. Lorenzani ${ }^{18}$, \\ G. Melnick ${ }^{21}$, B. Nisini ${ }^{25}$, S. Pacheco ${ }^{3}$, L. Pagani1 ${ }^{16}$, B. Parise ${ }^{17}$, J. Pearson ${ }^{19}$, T. Phillips ${ }^{13}$, M. Salez ${ }^{16}$, P. Saraceno ${ }^{14}$, \\ P. Schilke ${ }^{17,22}$, K. Schuster ${ }^{23}$, X. Tielens ${ }^{24}$, M. H. D. van der Wiel ${ }^{4,5}$, C. Vastel ${ }^{6,7}$, S. Viti $^{22}$, \\ V. Wakelam ${ }^{8,9}$, A. Walters ${ }^{6,7}$, F. Wyrowski ${ }^{17}$, H. Yorke ${ }^{19}$ P. Cais ${ }^{8}$, R. Güsten ${ }^{17}$, S. Philipp ${ }^{17}$, \\ T. Klein ${ }^{17}$, and F. Helmich ${ }^{4}$ \\ (Affiliations can be found after the references)
}

Received 31 May 2010 / Accepted 29 July 2010

\section{ABSTRACT}

In contrast with numerous studies on the physical and chemical structure of low- and high-mass protostars, much less is known about their intermediate-mass counterparts, a class of objects that could help to elucidate the mechanisms of star formation on both ends of the mass range. We present the first results from a rich HIFI spectral dataset on an intermediate-mass protostar, OMC2-FIR4, obtained in the CHESS (Chemical HErschel Survey of Star forming regions) key programme. The more than 100 methanol lines detected between 554 and $961 \mathrm{GHz}$ cover a range in upper level energy of 40 to $540 \mathrm{~K}$. Our physical interpretation focusses on the hot core, but likely the cold envelope and shocked regions also play a role in reality, because an analysis of the line profiles suggests the presence of multiple emission components. An upper limit of $10^{-6}$ is placed on the methanol abundance in the hot core, using a population diagram, large-scale source model and other considerations. This value is consistent with abundances previously seen in low-mass hot cores. Furthermore, the highest energy lines at the highest frequencies display asymmetric profiles, which may arise from infall around the hot core.

Key words. stars: formation - ISM: abundances - ISM: kinematics and dynamics - ISM: molecules

\section{Introduction}

Intermediate-mass, and therefore intermediate-luminosity protostars offer insights into the physical and chemical differences between the formation of low- and high-mass stars, but questions about their chemistry and dominant gas-heating mechanisms remain only partly answered.

Deep in the interior of a protostellar core, energy is released by a forming protostar. This energy heats the surrounding gas by dust-mediated and UV photon heating, as well as through shocks caused by protostellar outflows. The respective roles of these mechanisms as a function of protostar luminosity and the effects of heating on protostar evolution, are under intense study (e.g. Spaans et al. 1995; Doty et al. 2006; Bruderer et al. 2009), with the role of shocks and UV photons in low- and intermediate-luminosity sources emphasized by recent Herschel Space Observatory results (Fich et al. 2010; van Kempen et al. 2010). Sub-millimeter molecular line emission is a versatile probe of the physical and chemical conditions in these heated regions, the hot cores, revealing the initial conditions for

* Herschel is an ESA space observatory with science instruments provided by European-led principal Investigator consortia and with important participation from NASA. forming stars and planetary systems. Hot cores are compact (sizes $<0.1 \mathrm{pc}$ ), warm $(T>100 \mathrm{~K})$, and show evidence of complex chemistry (Kurtz et al. 2000; Ceccarelli et al. 2007). The two main paths to this complexity are gas-phase and grainsurface reactions (e.g. Garrod et al. 2008). During the gradual warm-up of grains in a hot core, species formed on them in earlier evolutionary phases react and the products are later released into the gas phase. Observable chemical differences include a methanol-formaldehyde abundance ratio, which increases with decreasing protostar luminosity (Cazaux et al. 2003; Bottinelli et al. 2004, 2007).

We present Herschel/HIFI sub-millimeter observations of methanol line emisson toward the intermediate-mass protostar OMC2-FIR4 ${ }^{1}$, attributing part of the emission to a hot core. With a luminosity of $1000 L_{\odot}$ (Crimier et al. 2009, hereafter Crim09) and a distance of only 440 pc (Hirota et al. 2007), the protostar OMC2-FIR4 is an excellent laboratory to study hot core chemistry in the intermediate mass regime. A structure model, constrained by $7.5^{\prime \prime}$ to $14.8^{\prime \prime}$ resolution dust-continuum maps and the broadband spectral energy distribution, was made for OMC2-FIR4 by Crim09.

${ }^{1}$ SIMBAD entry: [MWZ90] OMC-2 FIR 4. 
The uncertainties in the properties of the central component are large, but the model suggests a hot core radius of $R_{\text {Crim }}=$ 440 AU and densities above $\sim 5 \times 10^{6} \mathrm{~cm}^{-3}$. At $\sim 1^{\prime \prime}$ resolution, FIR4 is seen to consist of several clumps, which may be forming a cluster of protostars (Shimajiri et al. 2008, hereafter Shim08). Some of this activity may be triggered by an outflow from the nearby source OMC2-FIR3.

Methanol is a powerful diagnostic of the physical and chemical conditions in protostellar sources (van der Tak et al. 2000; Leurini et al. 2004, 2007; Maret et al. 2005; Wang et al. 2010), and is used to that end here. A careful analysis of methanol is also important for recovering the other species which $\mathrm{CH}_{3} \mathrm{OH}$ lines often blend with.

\section{Observations}

The source OMC2-FIR4 was observed with the HIFI spectrograph on the ESA Herschel Space Observatory (Pilbratt et al. 2010), using only the wide band spectrometer (WBS) (de Graauw et al. 2010), covering bands 1b (554.5 to $636.5 \mathrm{GHz}), 2 \mathrm{~b}(714.1$ to $801 \mathrm{GHz})$, and $3 \mathrm{~b}(858.0$ to 961.0 GHz). The WBS native resolution is $1.1 \mathrm{MHz}$. The data were taken in dual beam switch (DBS) spectral scan mode with a redundancy of four. More observations, expected later in 2010, will cover most of HIFI's frequency range. Median baseline subtraction and sideband deconvolution were performed with the HIPE 2 software (Ott 2010). A frequency-dependent main beam efficiency within three percent of 0.70 and a calibration uncertainty of $15 \%$ were used in calculating line fluxes. For a detailed review of HIFI's in-orbit performance, see Roelfsema et al. (2010).

The data quality in $1 \mathrm{~b}$ is superb, whereas $2 \mathrm{~b}$ and $3 \mathrm{~b}$ are significantly influenced by spurious features, "spurs", introduced by the local oscillator electronics, which act to increase noise in the single-sideband spectra and can damage spectral line profiles. Combining the $\mathrm{H}$ and $\mathrm{V}$ polarizations, the rms outside the bright lines is $T_{\mathrm{a}}^{\star}=10 \mathrm{mK}$ in $1 \mathrm{~b}, 21 \mathrm{mK}$ in $2 \mathrm{~b}$, and $26 \mathrm{mK}$ in $3 \mathrm{~b}$.

\section{Results}

Preliminary data processing reveals 91 lines from 17 species in band $1 \mathrm{~b}$, and similar numbers in $2 \mathrm{~b}$ and $3 \mathrm{~b}$, establishing OMC2FIR4 as a relatively line-rich protostar (see also the review by Ceccarelli et al. 2010). Methanol lines are advantageous to study in OMC2-FIR4 because they are abundant, cover a large range of excitation conditions, and are straightforward to measure because the spectrum is less crowded than in higher luminosity hot sources, in which more levels of more species can be significantly excited. Out of the hundreds of $\mathrm{CH}_{3} \mathrm{OH}$ transitions in each band, we detect 46 lines in band $1 \mathrm{~b}, 46$ in $2 \mathrm{~b}$, and 40 in $3 \mathrm{~b}$, with a range of $40 \mathrm{~K} \leq E_{\mathrm{u}} \leq 540 \mathrm{~K}$ in excitation energy. Identification made use of the JPL database ${ }^{2}$, with methanol data by Xu et al. (2008). This paper discusses lines detected with $>4 \sigma$ confidence and not blended or damaged by spurs.

\subsection{Line profiles}

Average line profiles were calculated in two energy ranges, $E_{\mathrm{u}} \leq 200 \mathrm{~K}$ and $E_{\mathrm{u}} \geq 200 \mathrm{~K}$, for each observed band. These six average profiles are presented in Fig. 1, with the number of lines and relevant beam sizes given. Before averaging, each line profile was resampled to an $0.2 \mathrm{~km} \mathrm{~s}^{-1}$ resolution grid with origin at the rest frequency, fitted by a single Gaussian and normalized by the intensity at the fit center. Distinguishing between the core $\left(|v|<2 \mathrm{~km} \mathrm{~s}^{-1}\right)$ and wings $\left(|v|>2 \mathrm{~km} \mathrm{~s}^{-1}\right)$ of a profile, we

2 The catalogue is available on-line at http://spec.jpl.nasa.gov

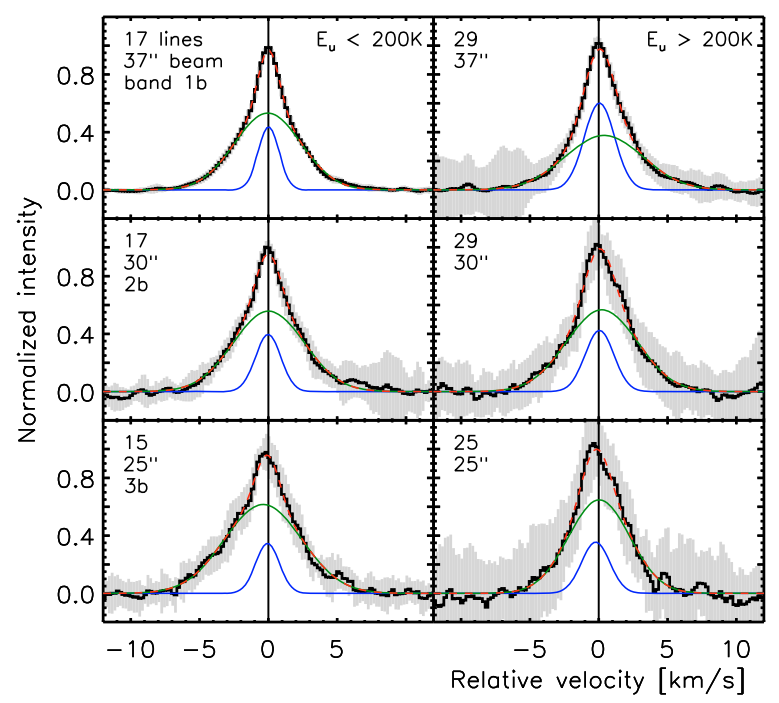

Fig. 1. Averaged $\mathrm{CH}_{3} \mathrm{OH}$ line profiles in the $E_{\mathrm{u}} \leq 200 \mathrm{~K}$ (left) and $E_{\mathrm{u}} \geq 200 \mathrm{~K}$ (right) ranges, for bands $1 \mathrm{~b}$ (top), 2b (middle) and $3 \mathrm{~b}$ (bottom). The gray area marks the biased weighted standard deviation of the sample of normalized profiles. The two fitted Gaussians (blue, green) and their sum (dashed red line) are given.

Table 1. Widths and central velocities of the two Gaussian fit components ("Fit") to each average line profile in Fig. 1.

\begin{tabular}{clc}
\hline \hline$E_{\mathrm{u}}$ range & Fit & $F W H M\left[\mathrm{~km} \mathrm{~s}^{-1}\right]($ bands $1 \mathrm{~b}, 2 \mathrm{~b}, 3 \mathrm{~b})$ \\
\hline$\leq 200 \mathrm{~K}$ & 1 & $(1.52,1.84,1.72) \pm(0.01,0.08,0.08)$ \\
& 2 & $(4.84,5.16,5.62) \pm(0.03,0.11,0.07)$ \\
\hline$\geq 200 \mathrm{~K}$ & 1 & $(2.22,2.08,2.06) \pm(0.04,0.11,0.28)$ \\
& 2 & $(5.68,5.16,4.44) \pm(0.13,0.13,0.24)$ \\
\hline \hline$E_{\mathrm{u}}$ range & Fit & Gaussian centre $\left[\mathrm{km} \mathrm{s}^{-1}\right]$ \\
\hline$\leq 200 \mathrm{~K}$ & 1 & $(0.02,-0.02,-0.06) \pm(0.01,0.03,0.04)$ \\
& 2 & $(-0.04,0.03,-0.38) \pm(0.01,0.04,0.04)$ \\
\hline$\geq 200 \mathrm{~K}$ & 1 & $(0.05,0.05,-0.22) \pm(0.02,0.04,0.10)$ \\
& 2 & $(0.39,0.23,0.04) \pm(0.05,0.05,0.10)$ \\
\hline
\end{tabular}

note that the cores of the average line profiles seem to show a broadening trend with decreasing beam size and increasing $E_{\mathrm{u}}$. Also, the wings are weaker in the higher energy regime than in the lower.

Two-component blind Gaussian fits were made for the average profiles in each energy range and band. The fits systematically separate into a narrow and broad component, the widths and central locations of which are given in Table 1 . The superiority of a two-Gaussian fit to that of a single Gaussian, as well as the trends mentioned in the previous paragraph, strongly suggest the presence of at least two physical emission components with different beam filling factors, as discussed in Sect. 4.

\subsection{Population diagram and LTE modelling}

The detection of $132 \mathrm{CH}_{3} \mathrm{OH}$ lines covering $500 \mathrm{~K}$ in upper level energy presents an excellent opportunity to perform a population diagram analysis (Goldsmith \& Langer 1999) to study the excitation conditions. In Fig. 2 we present the population diagram for the observed methanol lines. Line fluxes were obtained from single-Gaussian fits.

Part of the vertical scatter in Fig. 2 is due to optical depth, estimated from LTE modelling to contribute $\Delta \ln \left(N_{\mathrm{u}} / g_{\mathrm{u}}\right) \leq$ 0.37. Another factor is beam dilution, which contributes up to $\Delta \ln \left(N_{\mathrm{u}} / g_{\mathrm{u}}\right) \approx 2 \ln \left(37^{\prime \prime} / 25^{\prime \prime}\right) \approx 0.8$ for a point source observed 


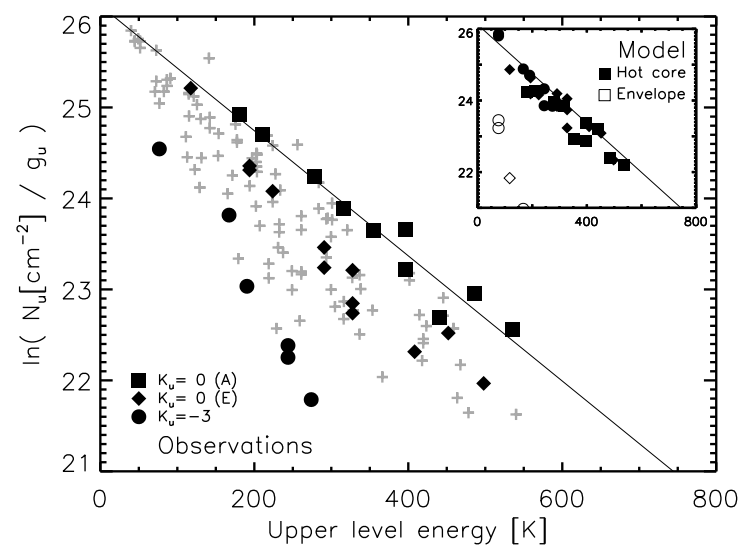

Fig. 2. Observed and modelled (inset) $\mathrm{CH}_{3} \mathrm{OH}$ population diagrams, plotting column density, divided by statistical weight, versus upper level energy for detected lines from all observed HIFI bands. Most transitions are given as crosses, but the $K_{\mathrm{u}}=0$ ladders for $A$ and $E$ states are indicated by filled squares and diamonds, and $K_{\mathrm{u}}=-3$ by filled circles. The inset gives the synthetic population diagram from our LTE model for $K_{\mathrm{u}}=0$ and -3 , with solid and open symbols representing the hot core and envelope contributions. The linear fit to the observed $K_{\mathrm{u}}=0$ $A$ state lines is shown on both plots.

at the spectral edges of bands $1 \mathrm{~b}$ and $3 \mathrm{~b}$. However, most of the scatter is due to differences in thermalization conditions for $\mathrm{CH}_{3} \mathrm{OH}$ transitions with different upper level $K$ quantum numbers. We assume the K-ladders with the lowest $n_{\mathrm{cr}}$, e.g. $K_{\mathrm{u}}=0$, approach LTE, as they thermalize below the densities expected for the hot core from the Crim09 model and are seen to lie close to each other, forming the upper envelope of the diagram. The $K_{\mathrm{u}}=-3$ ladder, which has a critical density of $n_{\mathrm{cr}} \approx 10^{9} \mathrm{~cm}^{-3}$, forms the lower envelope, well below most detected ladders $\left(-4 \leq K_{\mathrm{u}} \leq 4\right)$.

Excitation temperatures and column densities from linear fitting are given in Table 2 for the ladder likely closest to LTE, $K_{\mathrm{u}}=0$. The fit to the $A$ state is shown in Fig. 2 and yields $T_{\text {rot }}=(145 \pm 12) K$ and $N_{\mathrm{A}}=(2.2 \pm 0.5) \times 10^{14} \mathrm{~cm}^{-2}$. Fitting the $E$ state gives $T_{\text {rot }}=(120 \pm 8) \mathrm{K}$ and $N_{\mathrm{A}}=(1.4 \pm 0.3) \times 10^{14} \mathrm{~cm}^{-2}$. A fit to the $K_{\mathrm{u}}=-3$ ladder gives $T_{\text {rot }}=(66 \pm 2) \mathrm{K}$. This low value is likely mostly due to subthermal excitation, but optical depth and beam dilution may also play a role, as explained earlier.

A two-component LTE model was made with the CASSIS software $^{3}$, treating methanol as a single species. The model envelope and hot core have methanol abundances of $2 \times 10^{-9}$ and $10^{-6}$, line widths of $3 \mathrm{~km} \mathrm{~s}^{-1}$ and apparent diameters of $20.0^{\prime \prime}$ and $3.5^{\prime \prime}$, respectively. Source sizes, $\mathrm{H}_{2}$ column densities, and excitation temperatures were taken to be consistent with the Crim09 source model, as well as with the observed $T_{\text {rot }}$ values in Table 2, and are discussed in Sect. 4. Table 2 gives the column densities and excitation temperatures we used as input to the model and the simulated observables derived from it. The simulated population diagram, including the Herschel/HIFI beam efficiency, is shown as the inset in Fig. 2. Optical depth in the modelled lines is typically $0.01 \ldots 0.80$. The LTE conditions align transitions from all $K$-ladders, decreasing scatter in the simulated diagram.

The model was adjusted to reproduce the $K_{\mathrm{u}}=0$ lines, which led to a solution where the hot core dominates the line fluxes, as seen in the inset of Fig. 2. As the $T_{\text {rot }}$ values in Table 2 show, a linear fit to the simulated observations yields results different from the model input. This is due to optical depth, blending, and

${ }^{3}$ CASSIS has been developed by CESR-UPS/CNRS (http:// cassis. cesr.fr).
Table 2. Excitation temperature and column densities.

\begin{tabular}{rlll}
\hline \hline Approach & Component & $\mathrm{T}[\mathrm{K}]($ type $)$ & $N_{\text {meth }}\left[\mathrm{cm}^{-2}\right]$ \\
\hline Population & $K_{\mathrm{u}}=0(A)$ & $145 \pm 12(\mathrm{rot})$ & $(2.2 \pm 0.5) \times 10^{14}$ \\
diagram & $K_{\mathrm{u}}=0(E)$ & $120 \pm 8(\mathrm{rot})$ & $(1.4 \pm 0.3) \times 10^{14}$ \\
\hline LTE model & Envelope & $40(\mathrm{exc})$ & $2 \times 10^{14}$ \\
input & Hot core & $120(\mathrm{exc})$ & $6 \times 10^{16}$ \\
\hline LTE simu- & $K_{\mathrm{u}}=0(A)$ & $164(\mathrm{rot})$ & $1.4 \times 10^{14}$ \\
lated obs. & $K_{\mathrm{u}}=0(E)$ & $168(\mathrm{rot})$ & $1.7 \times 10^{14}$ \\
\hline
\end{tabular}

Notes. From the top: the rotational temperatures and methanol column densities derived from the observed population diagram; the input excitation temperatures and column densities of the LTE model; the rotational temperatures and column densities derived from the modelled population diagram.

beam dilution effects. The $T_{\text {rot }}$ derived from the simulation is sensitive to factor two changes in input line width at the $10 \%$ level due to optical depth effects. Owing to the high upper level energies of the detected $K_{\mathrm{u}}=0$ transitions, the envelope emission only influences the derived $T_{\text {rot }}$ at the $1 \%$ level in the model. It is not clear that LTE is a valid assumption even for the $K_{\mathrm{u}}=0$ ladder, and thus a more comprehensive, non-LTE modelling effort is underway. Preliminary results suggest the hot core density is higher than in the Crim09 best-fit model.

\section{Discussion}

The source OMC2-FIR4 is a line-rich protostar with a high degree of chemical and physical complexity. The hundreds of $\mathrm{CH}_{3} \mathrm{OH}$ transitions that are detectable with HIFI cover a wide range of excitation conditions and offer unprecedented spectral constraints on the structure of protostellar cores.

Previous knowledge (Jørgensen et al. 2006, Shim08, Crim09) suggests OMC2-FIR4 is dominated by three components: a large-scale, cool envelope $\sim 10^{4}$ AU across; a compact hot core $\sim 10^{2} \mathrm{AU}$ across; and the outflow from FIR3, in particular a suspected blue-shifted spot resulting from its interaction with the FIR4 envelope. The hot core gives rise to a dominant part of the emission in all lines in our LTE model, but the changes in line profiles through Fig. 1 imply reality is more complex. The envelope may contribute significantly to the lowest excitation lines, and shocks from regions such as where the FIR3 outflow strikes FIR4 (Shim08) are likely to be important.

The effects of the hot core radius and $\mathrm{H}_{2}$ column density on the line fluxes are degenerate if the lines are optically thin, as they are in our LTE model. Thus, an upper limit on the former and lower limit on the latter will result in an upper limit on the methanol abundance. Attributing the luminosity of FIR $4,10^{3} L_{\odot}$, to the central protostar and assuming grain mantle evaporation at $100 \mathrm{~K}$, one finds an upper limit of $R_{\text {core }} \approx 760 \mathrm{AU}$ for $0.1 \mu \mathrm{m}$ olivine grains. More realistic radiative transfer would decrease this value. At $440 \mathrm{pc}, 760 \mathrm{AU}$ extends $\sim 1.75^{\prime \prime}$ on the sky, which is adopted as the core radius in the model.

To reproduce the observed $K_{\mathrm{u}}=0$ line fluxes with LTE at an assumed $T_{\text {exc }}=120 \mathrm{~K}$, we need a column density of $N\left(\mathrm{CH}_{3} \mathrm{OH}\right)=6 \times 10^{16} \mathrm{~cm}^{-2}$ in the model hot core. Integrating the Crim09 $\mathrm{H}_{2}$ density distribution from 100 to $R_{\text {Crim }}=440 \mathrm{AU}$, one obtains $N\left(\mathrm{H}_{2}\right) \approx 6 \times 10^{22} \mathrm{~cm}^{-2}$. The source model was based on low-resolution maps, and the core is poorly constrained and excludes the inner $100 \mathrm{AU}$. We thus take the $\mathrm{H}_{2}$ column density as a lower limit and, adopting it in the LTE model, conclude that the obtained $\mathrm{CH}_{3} \mathrm{OH}$ abundance $\mathrm{X}_{\text {core }}=10^{-6}$ is a conservative upper limit. The highest abundance seen in low-mass hot cores is $2 \times 10^{-6}$ (Jørgensen et al. 2005). If the hot core does not 
dominate the line emission in reality, smaller hot core sizes and methanol column densities, as well as higher rotational temperatures, may be consistent with the data. Presently, the abundance of methanol in the envelope is very poorly constrained. We set it to $2 \times 10^{-9}$ in the LTE model, consistent with the factor $10^{2 \ldots 3}$ abundance jump seen in hot cores (van der Tak et al. 2000; Maret et al. 2005).

The hot core density is broadly constrained to be between $10^{6}$ and $10^{8} \mathrm{~cm}^{-3}$ by this early analysis. Lower and higher values would be difficult to reconcile with the large-scale density profile, furthermore the population diagram suggests the $K$ ladders with the lowest $n_{\mathrm{cr}}$ may approach LTE while those with the highest $n_{\text {cr }}$ clearly deviate from it. Typical kinetic temperatures well in the $\geq 100 \mathrm{~K}$ hot core regime are implied by the obtained rotational temperatures of $T_{\text {rot }} \approx(120 \ldots 145) \mathrm{K}$. Shock contributions from the FIR3-FIR4 interaction spot as well as the hot core itself need to be considered before drawing further conclusions about the temperature structure.

An intriguing feature is the appearance of an asymmetry in the $E_{\mathrm{u}} \geq 200 \mathrm{~K}$ lines, as seen in Fig. 1. Because these lines should be dominated by the hot core emission, the profiles observed could represent the blue asymmetric profile expected for infalling gas (e.g. Walker et al. 1994), implying we may be seeing collapse in the central regions. To clarify whether infall, shocks, or other phenomena are responsible for the asymmetry, interferometry as well as further analysis of the Herschel/HIFI data, in particular the ${ }^{13} \mathrm{CH}_{3} \mathrm{OH}$ lines, will be employed.

We note the difference in column density of the $K_{\mathrm{u}}=0$ transitions of the $A$ and $E$ states in Table 2. Whether it is truly significant will be explored in a future paper. Also, early results from an analysis of formaldehyde in OMC2-FIR4 will be presented in a companion paper (Crimier et al., in prep.).

Acknowledgements. The authors are grateful to the referee, Dr. Tim van Kempen, for constructive comments leading to a significant improvement of the paper, and to Rens Waters for helpful discussions. HIFI has been designed and built by a consortium of institutes and university departments from across Europe, Canada and the United States under the leadership of SRON Netherlands Institute for Space Research, Groningen, The Netherlands and with major contributions from Germany, France and the US. Consortium members are: Canada: CSA, U.Waterloo; France: CESR, LAB, LERMA, IRAM; Germany: KOSMA, MPIfR, MPS; Ireland, NUI Maynooth; Italy: ASI, IFSI-INAF, Osservatorio Astrofisico di Arcetri- INAF; Netherlands: SRON, TUD; Poland: CAMK, CBK; Spain: Observatorio AstronŮmico Nacional (IGN), Centro de Astrobiologia (CSIC-INTA). Sweden: Chalmers University of Technology - MC2, RSS \& GARD; Onsala Space Observatory; Swedish National Space Board, Stockholm University - Stockholm Observatory; Switzerland: ETH Zurich, FHNW; USA: Caltech, JPL, NHSC, and we are deeply grateful to everyone involved in the designing, building, and exploitation of this fantastic instrument. HCSS, HSpot, and HIPE are joint developments by the Herschel Science Ground Segment Consortium, consisting of ESA, the NASA Herschel Science Center, and the HIFI, PACS, and SPIRE consortia. M.Kama gratefully acknowledges support from the Netherlands Organisation for Scientific Research (NWO) grant number 021.002.081 and the Leids Kerkhoven-Bosscha Fonds, and thanks SRON Groningen for hosting the HIFI ICC volunteers.

\section{References}

Bottinelli, S., Ceccarelli, C., Lefloch, B., et al. 2004, ApJ, 615, 354

Bottinelli, S., Ceccarelli, C., Williams, J. P., \& Lefloch, B. 2007, A\&A, 463, 601 Bruderer, S., Benz, A. O., Doty, S. D., van Dishoeck, E. F., \& Bourke, T. L. 2009, ApJ, 700, 872

Cazaux, S., Tielens, A. G. G. M., Ceccarelli, C., et al. 2003, ApJ, 593, L51

Ceccarelli, C., Caselli, P., Herbst, E., Tielens, A. G. G. M., \& Caux, E. 2007, Protostars and Planets V, 47
Ceccarelli, C., Bacmann, A., Boogert, A., et al., 2010, A\&A, 521, L22 Crimier, N., Ceccarelli, C., Lefloch, B., \& Faure, A. 2009, A\&A, 506, 1229 de Graauw, Th., Helmich, F. P., Phillips, T. G., et al. 2010, A\&A, 518, L6 Doty, S. D., van Dishoeck, E. F., \& Tan, J. C. 2006, A\&A, 454, L5 Fich, M., Johnstone, D., van Kempen, T. A., et al. 2010, A\&A, 518, L86+ Garrod, R. T., Weaver, S. L. W., \& Herbst, E. 2008, ApJ, 682, 283 Goldsmith, P. F., \& Langer, W. D. 1999, ApJ, 517, 209

Hirota, T., Bushimata, T., Choi, Y. K., et al. 2007, PASJ, 59, 897

Jørgensen, J. K., Bourke, T. L., Myers, P. C., et al. 2005, ApJ, 632, 973

Jørgensen, J. K., Johnstone, D., van Dishoeck, E. F., \& Doty, S. D. 2006, A\&A, 449,609

Kurtz, S., Cesaroni, R., Churchwell, E., Hofner, P., \& Walmsley, C. M. 2000, Protostars and Planets IV, 299

Leurini, S., Schilke, P., Menten, K. M., et al. 2004, A\&A, 422, 573

Leurini, S., Schilke, P., Wyrowski, F., \& Menten, K. M. 2007, A\&A, 466, 215

Maret, S., Ceccarelli, C., Tielens, A. G. G. M., et al. 2005, A\&A, 442, 527

Ott, S. 2010, in Astronomical Data Analysis Software and Systems XIX, ed. Y. Mizumoto, \& M. Ohishi, ASP Conf. Ser., in press

Pilbratt, G. L., Riedinger, J. R., Passvogel, T., et al. 2010, A\&A, 518, L1

Roelfsema, P. R., Helmich, F. P., Teyssier, D., et al. 2010, A\&A, submitted

Shimajiri, Y., Takahashi, S., Takakuwa, S., Saito, M., \& Kawabe, R. 2008, ApJ, 683,255

Spaans, M., Hogerheijde, M. R., Mundy, L. G., \& van Dishoeck, E. F. 1995, ApJ, 455, L167

van der Tak, F. F. S., van Dishoeck, E. F., \& Caselli, P. 2000, A\&A, 361, 327

van Kempen, T. A., Kristensen, L. E., Herczeg, G. J., et al. 2010, A\&A, 518, L121

Walker, C. K., Narayanan, G., \& Boss, A. P. 1994, ApJ, 431, 767

Wang, S., Bergin, E. A., Crockett, N. R., et al. 2010, A\&A, submitted

Xu, L., Fisher, J., Lees, R. M., et al. 2008, J. Mol. Spectr., 251, 305

1 Astronomical Institute "Anton Pannekoek", University of Amsterdam, Amsterdam, The Netherlands

2 Department of Astrophysics/IMAPP, Radboud University Nijmegen, Nijmegen, The Netherlands

3 Laboratoire d'Astrophysique de Grenoble, UMR 5571-CNRS, Université Joseph Fourier, Grenoble, France

4 SRON Netherlands Institute for Space Research, Groningen, The Netherlands

5 Kapteyn Astronomical Institute, University of Groningen, The Netherlands

6 Centre d'Étude Spatiale des Rayonnements, Université Paul Sabatier, Toulouse, France

7 CNRS/INSU, UMR 5187, Toulouse, France

8 Université de Bordeaux, Laboratoire d'Astrophysique de Bordeaux, Floirac, France

9 CNRS/INSU, UMR 5804, Floirac Cedex, France

10 IGN Observatorio Astronómico Nacional, Alcalá de Henares, Spain

11 Centro de Astrobiologìa, CSIC-INTA, Madrid, Spain

12 Infared Processing and Analysis Center, Caltech, Pasadena, USA

13 California Institute of Technology, Pasadena, USA

14 INAF - Istituto di Fisica dello Spazio Interplanetario, Roma, Italy

15 School of Physics and Astronomy, University of Leeds, Leeds, UK

16 Laboratoire d'Études du Rayonnement et de la Matière en Astrophysique, UMR 8112 CNRS/INSU, OP, ENS, UPMC, UCP, Paris, France

17 Max-Planck-Institut für Radioastronomie, Bonn, Germany

18 INAF Osservatorio Astrofisico di Arcetri, Florence, Italy

19 Jet Propulsion Laboratory, Caltech, Pasadena, CA 91109, USA

20 Ohio State University, Columbus, OH, USA

21 Harvard-Smithsonian Center for Astrophysics, Cambridge MA, USA

22 Physikalisches Institut, Universität zu Köln, Köln, Germany

23 Institut de RadioAstronomie Millimétrique, Grenoble, France

24 Leiden Observatory, Leiden University, Leiden, The Netherlands

25 INAF - Osservatorio Astronomico di Roma, Monte Porzio Catone, Italy

26 Department of Astronomy, University of Michigan, Ann Arbor, USA

27 Max-Planck-Institut für Astronomie, Heidelberg, Germany 